\title{
A new approach to Mannheim curve in Euclidean
}

\section{3-space}

\author{
Ali Uçum, Çetin Camcı and Kazım İlarslan
}

\begin{abstract}
In this article, a new approach is given for Mannheim curves in 3dimensional Euclidean space. Thanks to this approach, the necessary and sufficient conditions including the known results have been obtained for a curve to be Mannheim curve in $\mathbb{E}^{3}$. In addition, related examples and graphs are given by showing that Salkowski and anti-Salkowski curves can be the examples of Mannheim curves and their mates. Finally, the Mannheim partner curves are characterized in $\mathbb{E}^{3}$.
\end{abstract}

Keywords. Mannheim curves, general helices, anti-Salkowski curves, Euclidean 3-space.

\section{Introduction}

In the Euclidean 3-space $\mathbb{E}^{3}$, a curve is called a general helix if its tangent vector makes a constant angle with a fixed straight line (the axis of the general helix). According to well-known result stated by M. A. Lancret in 1802 and first proved by B. de Saint Venant in 1845 (for details see $[4,5]$ ) a regular curve is a general helix if and only if the ratio of curvature to torsion is constant. Also it is known that a curve is called a circular helix if both curvatures $k_{1}$ and $k_{2}$ are non-zero constant. Circular helices geometrically appear as geodesic in right cylinders shaped on circle. The geodesics of a right cylinder, with arbitrary cross section, are called general or Lancret helices. In addition, Izumiya and Takeuchi have introduced the concept of slant helix having a property that the normal lines make a constant angle with a fixed straight line. They characterize a slant helix by the necessary and the suffcient condition that the geodesic curvature

$$
\kappa_{g}=\left(\frac{\kappa_{2}}{\kappa_{1}}\right)^{\prime} \frac{\kappa_{1}^{2}}{\left(\kappa_{1}^{2}+\kappa_{2}^{2}\right)^{3 / 2}}
$$

of spherical image of its principal normal indicatrix is a constant function [3]. A family of curves with constant curvature but non-constant torsion is called Salkowski curves and a family of curves with constant torsion but non-constant curvature is called anti-Salkowski curves ([1], [9]). In [8], Monterde studied some of characterizations of these curves and he proved that their principal normal vector makes a constant angle with fixed straight line. So that Salkowski and anti-Salkowski curves are important examples of slant helices.

Received date: January 15, 2021; Published online: November 10, 2021. 2010 Mathematics Subject Classification. 53A04..

Corresponding author: Ali Uçum. 
On the other hand, there exist some kinds of associated curves whose the Frenet frame fields satisfy certain geometric conditions in the Euclidean space $\mathbb{E}^{3}$. An example of such curves are called Mannheim curves, which have a property that their principal normal lines coincide with the binormal lines of the Mannheim mate (partner) curve at the corresponding points of the curves. It is known that the curvature functions of Mannheim curve in $\mathbb{E}^{3}$ satisfy the equality $\kappa_{1}=a\left(\kappa_{1}^{2}+\kappa_{2}^{2}\right)$ for some positive constant number $a$ and its parametric equation is obtained in [6] (see also [7],[11]). Some characterizations of Mannheim curves in $\mathbb{E}^{3}$ can be found in [4] and $[6]$.

Let $\beta$ be a Mannheim curve and $\beta^{*}$ be the Mannheim partner curve of $\beta$ in Euclidean 3 -space $\mathbb{E}^{3}$. In the literature, $\beta^{*}$ is given by

$$
\beta^{*}(f(s))=\beta(s)+\lambda(s) N(s)
$$

where $N(s)$ is the principal normal vector field of $\beta$ and $f$ is a differentiable function [4]. With respect to (1.1), the vector $\overrightarrow{\beta^{*} \beta}$ must be parallel to $N$.

In this paper, following [2], we claim that the vector $\overrightarrow{\beta^{*} \beta}$ does not have to be parallel to $N$, which is a special case for choosing the Mannheim partner curve. So we consider that the Mannheim partner curve of $\beta^{*}$ is given by

$$
\beta^{*}(f(s))=\beta(s)+u(s) T(s)+v(s) N(s)+w(s) B(s)
$$

where $\{T(s), N(s), B(s)\}$ is Frenet frame of $\beta$. Here if we take $u=w=0$, we obtain the case is used in the literature. So we give the generalization of Mannheim curves in $\mathbb{E}^{3}$. In this paper, we obtain the necessary and sufficient conditions for a curve in $\mathbb{E}^{3}$ to be Mannheim curve and give the related examples with respect to this new approach to Mannheim curves. Finally, we characterize the Mannheim partner curves in $\mathbb{E}^{3}$.

\section{New approach to Mannheim curves in Euclidean 3-space $\mathbb{E}^{3}$}

In this section, we will reconsider the Mannheim curves in Euclidean 3-space $\mathbb{E}^{3}$. Also in this paper, we will consider the case where $\kappa_{2} \neq 0$ so that the curve can lie fully in $\mathbb{E}^{3}$.

Definition 1. A curve $\beta: I \rightarrow \mathbb{E}^{3}$ with non-zero curvatures is a Mannheim curve if there is a curve $\beta^{*}: I^{*} \rightarrow \mathbb{E}^{3}$ such that the principal normal vectors of $\beta(s)$ coincide with the binormal vectors of $\beta^{*}\left(s^{*}\right)$ at $s \in I, s^{*} \in I^{*}$. In this case, $\beta^{*}\left(s^{*}\right)$ is called the Mannheim partner curve of $\beta(s)$.

Let $\beta: I \rightarrow \mathbb{E}^{3}$ be a Mannheim curve in $\mathbb{E}^{3}$ with the Frenet frame $\{T, N, B\}$ and the non-zero curvatures $\kappa_{1}, \kappa_{2}$, and $\beta^{*}: I^{*} \rightarrow \mathbb{E}^{3}$ be a Mannheim partner curve of $\beta$ with the Frenet frame $\left\{T^{*}, N^{*}, B^{*}\right\}$ and the non-zero curvatures $\kappa_{1}^{*}, \kappa_{2}^{*}$. Then $\beta^{*}$ can be written as

$$
\beta^{*}\left(s^{*}\right)=\beta^{*}(f(s))=\beta(s)+u(s) T(s)+v(s) N(s)+w(s) B(s)
$$

where $u(s), v(s)$ and $w(s)$ are differentiable functions on $I$.

Theorem 2.1. Let $\beta: I \subset \mathbb{R} \rightarrow \mathbb{E}^{3}$ be a unit speed curve with the non-zero curvatures $\kappa_{1}, \kappa_{2}$. Then the curve $\beta$ is a Mannheim curve with Mannheim partner $\beta^{*}$ if and only if there exist differentiable functions $u, v, w$ satisfying

$$
u \kappa_{1}+v^{\prime}=w \kappa_{2}, \quad w^{\prime}+v \kappa_{2} \neq 0, \quad\left(1+u^{\prime}-v \kappa_{1}\right) \kappa_{1}=\left(w^{\prime}+v \kappa_{2}\right) \kappa_{2} .
$$


Proof. Assume that $\beta$ is a Mannheim curve parametrized by arc-length $s$ with non-zero curvatures $\kappa_{1}, \kappa_{2}$ and the curve $\beta^{*}$ is the Mannheim partner curve of the curve $\beta$ parametrized by with arclength or pseudo arc $s^{*}$. Then, we can write the curve $\beta^{*}$ as

$$
\beta^{*}\left(s^{*}\right)=\beta^{*}(f(s))=\beta(s)+u(s) T(s)+v(s) N(s)+w(s) B(s)
$$

for all $s \in I$ where $u(s), v(s)$ and $w(s)$ are differentiable functions on $I$. Differentiating (2.2) with respect to $s$, we get

$$
T^{*} f^{\prime}=\left(1+u^{\prime}-v \kappa_{1}\right) T+\left(u \kappa_{1}+v^{\prime}-w \kappa_{2}\right) N+\left(w^{\prime}+v \kappa_{2}\right) B .
$$

By taking the scalar product of (2.3) with $N$, we have

$$
u \kappa_{1}+v^{\prime}-w \kappa_{2}=0 .
$$

Substituting (2.4) in (2.3), we find

$$
T^{*} f^{\prime}=\left(1+u^{\prime}-v \kappa_{1}\right) T+\left(w^{\prime}+v \kappa_{2}\right) B .
$$

By taking the scalar product of (2.5) with itself, we obtain

$$
\left(f^{\prime}\right)^{2}=\left(1+u^{\prime}-v \kappa_{1}\right)^{2}+\left(w^{\prime}+v \kappa_{2}\right)^{2} .
$$

If we denote

$$
\delta=\frac{1+u^{\prime}-v \kappa_{1}}{f^{\prime}} \text { and } \quad \gamma=\frac{w^{\prime}+v \kappa_{2}}{f^{\prime}}
$$

we get

$$
T^{*}=\delta T+\gamma B
$$

Differentiating (2.8) with respect to $s$, we find

$$
f^{\prime} \kappa_{1}^{*} N^{*}=\delta^{\prime} T+\left(\delta \kappa_{1}-\gamma \kappa_{2}\right) N+\gamma^{\prime} B .
$$

By taking the scalar product of (2.9) with $N$, we get $\delta \kappa_{1}-\gamma \kappa_{2}=0$, which implies that

$$
\left(1+u^{\prime}-v \kappa_{1}\right) \kappa_{1}=\left(w^{\prime}+v \kappa_{2}\right) \kappa_{2}
$$

where $w^{\prime}+v \kappa_{2} \neq 0$.

Conversely, assume that $\beta$ is a curve parametrized by arc-length $s$ with non-zero curvatures $\kappa_{1}, \kappa_{2}$ and the conditions of (2.1) hold for differentiable functions $u, v, w$. Then, we can define a curve $\beta^{*}$ as

$$
\beta^{*}\left(s^{*}\right)=\beta(s)+u(s) T(s)+v(s) N(s)+w(s) B(s) .
$$

Differentiating (2.11) with respect to $s$, we find

$$
\frac{d \beta^{*}}{d s}=\left(1+u^{\prime}-v \kappa_{1}\right) T+\left(w^{\prime}+v \kappa_{2}\right) B .
$$

which leads to that

$$
f^{\prime}=\sqrt{\left\langle\frac{d \beta^{*}}{d s}, \frac{d \beta^{*}}{d s}\right\rangle}=\frac{m_{1}\left(w^{\prime}+v \kappa_{2}\right) \sqrt{\kappa_{1}^{2}+\kappa_{2}^{2}}}{\kappa_{1}}
$$


where $m_{1}=\operatorname{sgn}\left(w^{\prime}+v \kappa_{2}\right)$. Rewriting (2.12), we obtain

$$
T^{*}=\frac{m_{1}}{\sqrt{\kappa_{1}^{2}+\kappa_{2}^{2}}}\left(\kappa_{2} T+\kappa_{1} B\right), \quad g\left(T^{*}, T^{*}\right)=1 .
$$

If we put

$$
\lambda_{1}=\frac{m_{1} \kappa_{2}}{\sqrt{\kappa_{1}^{2}+\kappa_{2}^{2}}} \quad \text { and } \quad \lambda_{2}=\frac{m_{1} \kappa_{1}}{\sqrt{\kappa_{1}^{2}+\kappa_{2}^{2}}}
$$

we get

$$
T^{*}=\lambda_{1} T+\lambda_{2} B
$$

Differentiating (2.15) with respect to $s$, we find

$$
\frac{d T^{*}}{d s^{*}}=\frac{\lambda_{1}^{\prime}}{f^{\prime}} T+\frac{\lambda_{2}^{\prime}}{f^{\prime}} B
$$

which cause that

$$
\kappa_{1}^{*}=\left\|\frac{d T^{*}}{d s^{*}}\right\|=\frac{\sqrt{\left(\lambda_{1}^{\prime}\right)^{2}+\left(\lambda_{2}^{\prime}\right)^{2}}}{f^{\prime}}=\frac{m_{2}\left(\kappa_{2} \kappa_{1}^{\prime}-\kappa_{1} \kappa_{2}^{\prime}\right)}{f^{\prime}\left(\kappa_{1}^{2}+\kappa_{2}^{2}\right)}=\frac{-m_{2} \kappa_{1}^{2}\left(\frac{\kappa_{2}}{\kappa_{1}}\right)^{\prime}}{f^{\prime}\left(\kappa_{1}^{2}+\kappa_{2}^{2}\right)},
$$

where $m_{2}=\operatorname{sgn}\left(\kappa_{2} \kappa_{1}^{\prime}-\kappa_{1} \kappa_{2}^{\prime}\right)$. Now, we can find $N^{*}$ as

$$
N^{*}=\frac{m_{1} m_{2}}{\sqrt{\kappa_{1}^{2}+\kappa_{2}^{2}}}\left(-\kappa_{1} T+\kappa_{2} B\right), \quad g\left(N^{*}, N^{*}\right)=1 .
$$

Now, we define $B^{*}$ as

$$
B^{*}=T^{*} \times N^{*}=-m_{2} N, \quad g\left(B^{*}, B^{*}\right)=1 .
$$

Lastly we find

$$
\kappa_{2}^{*}=-\left\langle\frac{d B^{*}}{d s^{*}}, N^{*}\right\rangle=\frac{m_{1} \sqrt{\kappa_{1}^{2}+\kappa_{2}^{2}}}{f^{\prime}} \neq 0 .
$$

Then $\beta^{*}$ is a Mannheim partner curve of $\beta$. Thus $\beta$ is a Mannheim curve.

If we take $u=w=0$ in Theorem 2.1, we get the conditions of classical Mannheim curves in the literature with the Mannheim partner curve $\beta^{*}$ given by

$$
\beta^{*}(s)=\beta(s)+v(s) N(s) .
$$

Corollary 2.2. Let $\beta: I \subset \mathbb{R} \rightarrow \mathbb{E}^{3}$ be a unit speed curve with the non-zero curvatures $\kappa_{1}, \kappa_{2}$. Then the curve $\beta$ is a Mannheim curve with Mannheim partner $\beta^{*}$ given by

$$
\beta^{*}(s)=\beta(s)+v(s) N(s)
$$

if and only if there exist real number $v$ satisfying $\kappa_{1}=v\left(\kappa_{1}^{2}+\kappa_{2}^{2}\right)$.

Corollary 2.3. Let $\beta: I \subset \mathbb{R} \rightarrow \mathbb{E}^{3}$ be a general helix with the non-zero curvatures $\kappa_{1}, \kappa_{2}$. Then $\beta$ does not have the Mannheim partner curve liying fully in $\mathbb{E}^{3}$ (or the Mannheim partner curve is a straight line).

Proof. Assume that $\beta: I \subset \mathbb{R} \rightarrow \mathbb{E}^{3}$ is a Mannheim general helix with the non-zero curvatures $\kappa_{1}, \kappa_{2}$. Then the ratio $\kappa_{2} / \kappa_{1}$ is constant which implies from $(2.16)$ that $\kappa_{1}^{*}=0$. Then $\beta^{*}$ is a straight line. 
Corollary 2.4. Let $\beta: I \subset \mathbb{R} \rightarrow \mathbb{E}^{3}$ be a Mannheim curve with the curvatures $\kappa_{1}, \kappa_{2}$ and the curve $\beta^{*}$ be a Mannheim partner curve of $\beta$ with the curvatures $\kappa_{1}^{*}, \kappa_{2}^{*}$. Then $\beta^{*}$ is a general helix if and only if $\beta$ is a slant helix.

Proof. Assume that $\beta: I \subset \mathbb{R} \rightarrow \mathbb{E}^{3}$ is a Mannheim curve with the curvatures $\kappa_{1}, \kappa_{2}$ and the curve $\beta^{*}$ is a Mannheim partner curve of $\beta$. Then from (2.16) and (2.18), we have

$$
\frac{\kappa_{1}^{*}}{\kappa_{2}^{*}}=-m_{1} m_{2}\left(\frac{\kappa_{2}}{\kappa_{1}}\right)^{\prime} \frac{\kappa_{1}^{2}}{\left(\kappa_{1}^{2}+\kappa_{2}^{2}\right)^{3 / 2}} .
$$

So $\beta^{*}$ is a general helix if and only if $\beta$ is a slant helix.

In the following example, we give a Mannheim partner curve of a Mannheim curve in $\mathbb{E}^{3}$.

Example 1. Let $\beta: I \subset \mathbb{R} \rightarrow \mathbb{E}^{3}$ be a Mannheim curve with the curvatures $\kappa_{1}, \kappa_{2}$. Then the conditions of Theorem 2.1 are satisfied. Assume that $v=v_{0} \in \mathbb{R}$. Then we can find

$$
u \kappa_{1}=w \kappa_{2} \quad \text { and } \quad\left(1+u^{\prime}-v_{0} \kappa_{1}\right) \kappa_{1}=\left(w^{\prime}+v_{0} \kappa_{2}\right) \kappa_{2},
$$

which implies that

$$
w=\frac{v_{0}\left(\kappa_{1}^{2}+\kappa_{2}^{2}\right)-\kappa_{1}}{\kappa_{1}\left(\frac{\kappa_{2}}{\kappa_{1}}\right)^{\prime}} \text { and } u=\frac{\kappa_{2}\left(v_{0}\left(\kappa_{1}^{2}+\kappa_{2}^{2}\right)-\kappa_{1}\right)}{\kappa_{1}^{2}\left(\frac{\kappa_{2}}{\kappa_{1}}\right)^{\prime}} .
$$

Thus we obtain the Mannheim partner curve $\beta^{*}$ as

$$
\beta^{*}=\beta+\frac{\kappa_{2}\left(v_{0}\left(\kappa_{1}^{2}+\kappa_{2}^{2}\right)-\kappa_{1}\right)}{\kappa_{1}^{2}\left(\frac{\kappa_{2}}{\kappa_{1}}\right)^{\prime}} T+v_{0} N+\frac{v_{0}\left(\kappa_{1}^{2}+\kappa_{2}^{2}\right)-\kappa_{1}}{\kappa_{1}\left(\frac{\kappa_{2}}{\kappa_{1}}\right)^{\prime}} B .
$$

In the following example, we give an example for a Salkowski curve which is Mannheim curve. This example is new in the literature.

Example 2. Let us consider the Salkowski curve in $\mathbb{E}^{3}$ given by

$$
\beta(s)=\left(\begin{array}{l}
\frac{78 s \sqrt{25-s^{2}} \cos \left(\sqrt{26} \arcsin \left(\frac{s}{5}\right)\right)+\sqrt{26}\left(28 s^{2}-625\right) \sin \left(\sqrt{26} \arcsin \left(\frac{s}{5}\right)\right)}{2860}, \\
\frac{\sqrt{26}\left(625-28 s^{2}\right) \cos \left(\sqrt{26} \arcsin \left(\frac{s}{5}\right)\right)+78 s \sqrt{25-s^{2}} \sin \left(\sqrt{26} \arcsin \left(\frac{s}{5}\right)\right)}{2860}, \\
\frac{25-2 s^{2}}{4 \sqrt{26}}
\end{array}\right)
$$

with the curvatures $\kappa_{1}=1$ and $\kappa_{2}=s / \sqrt{25-s^{2}}$ and the Frenet frame as

$$
\begin{aligned}
T & =\left(\begin{array}{l}
\frac{-\sqrt{25-s^{2}} \cos \left(\sqrt{26} \arcsin \left(\frac{s}{5}\right)\right)}{5}-\frac{s \sin \left(\sqrt{26} \arcsin \left(\frac{s}{5}\right)\right)}{5 \sqrt{26}}, \\
\frac{s \cos \left(\sqrt{26} \arcsin \left(\frac{s}{5}\right)\right)}{5 \sqrt{26}}-\frac{\sqrt{25-s^{2}} \sin \left(\sqrt{26} \arcsin \left(\frac{s}{5}\right)\right)}{5}, \\
\left.-\frac{2}{\sqrt{26}}\right)^{2}
\end{array}\right), \\
N & =\left(\frac{5 \sin \left(\sqrt{26} \arcsin \left(\frac{s}{5}\right)\right)}{\sqrt{26}},-\frac{5 \cos \left(\sqrt{26} \arcsin \left(\frac{s}{5}\right)\right)}{\sqrt{26}},-\frac{1}{\sqrt{26}}\right), \\
B & =\left(\begin{array}{l}
\frac{s \cos \left(\sqrt{26} \arcsin \left(\frac{s}{5}\right)\right)}{5}-\frac{\sqrt{25-s^{2}} \sin \left(\sqrt{26} \arcsin \left(\frac{s}{5}\right)\right)}{5 \sqrt{26}}, \\
\frac{\sqrt{25-s^{2}} \cos \left(\sqrt{26} \arcsin \left(\frac{s}{5}\right)\right)}{5 \sqrt{26}}+\frac{s \sin \left(\sqrt{26} \arcsin \left(\frac{s}{5}\right)\right)}{5}, \\
-\frac{\sqrt{26}}{\sqrt{26}}
\end{array}\right) .
\end{aligned}
$$


If we take $v_{0}=0$ in Example 1, we obtain the Mannheim partner curve $\beta^{*}$ as follows

$$
\beta^{*}(s)=\left(\begin{array}{l}
\frac{3\left(26 s \sqrt{25-s^{2}} \cos \left(\sqrt{26} \arcsin \left(\frac{s}{5}\right)\right)+\sqrt{26}\left(2 s^{2}-25\right) \sin \left(\sqrt{26} \arcsin \left(\frac{s}{5}\right)\right)\right)}{2860}, \\
\frac{3 \sqrt{26}\left(25-2 s^{2}\right) \cos \left(\sqrt{26} \arcsin \left(\frac{s}{5}\right)\right)+78 s \sqrt{25-s^{2}} \sin \left(\sqrt{26} \arcsin \left(\frac{s}{5}\right)\right)}{2860}, \\
\frac{125-6 s^{2}}{4 \sqrt{26}}
\end{array}\right),
$$

with the curvatures

$$
\kappa_{1}^{*}=\frac{5}{3 s \sqrt{25-s^{2}}} \quad \text { and } \quad \kappa_{2}^{*}=\frac{25}{3 s \sqrt{25-s^{2}}}
$$

the Frenet frame as

$$
\begin{aligned}
T^{*} & =\left(-\frac{\sin \left(\sqrt{26} \arcsin \left(\frac{s}{5}\right)\right)}{\sqrt{26}}, \frac{\cos \left(\sqrt{26} \arcsin \left(\frac{s}{5}\right)\right)}{\sqrt{26}},-\frac{5}{\sqrt{26}}\right), \\
N^{*} & =\left(-\cos \left(\sqrt{26} \arcsin \left(\frac{s}{5}\right)\right),-\sin \left(\sqrt{26} \arcsin \left(\frac{s}{5}\right)\right), 0\right), \\
B^{*} & =\left(\frac{5 \sin \left(\sqrt{26} \arcsin \left(\frac{s}{5}\right)\right)}{\sqrt{26}},-\frac{5 \cos \left(\sqrt{26} \arcsin \left(\frac{s}{5}\right)\right)}{\sqrt{26}},-\frac{1}{\sqrt{26}}\right) .
\end{aligned}
$$

It can be easily obtained $N=B^{*}$ which implies that $\beta$ is a Mannheim curve whose Mannheim partner curve is $\beta^{*}$. Here $\beta^{*}$ is a general helix.
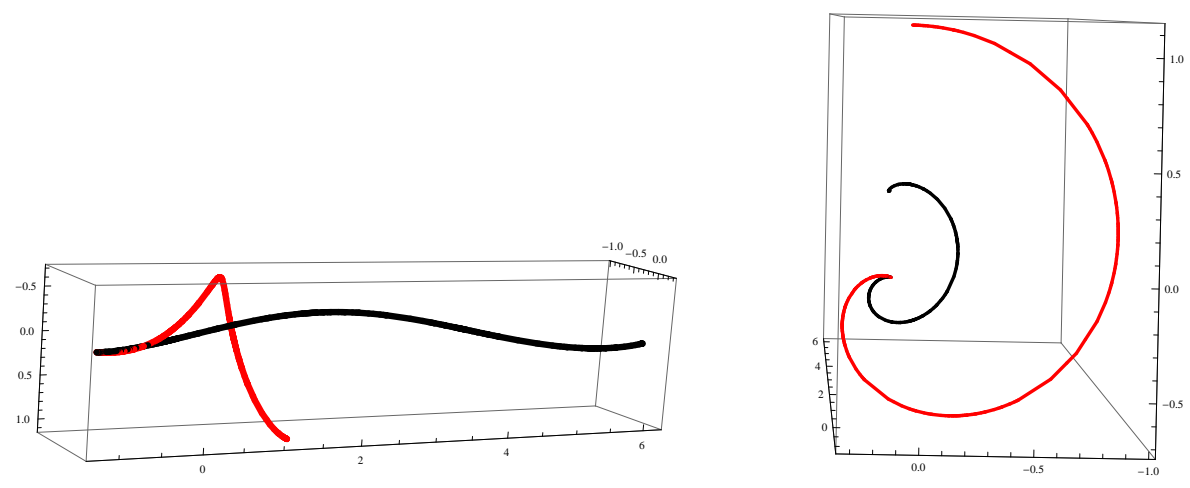

Figure 1: The figure contains same graphics from two different aspects. The red graphic is $\beta$ and the black graphic is $\beta^{*}$ in Example 2. (For interpretation of the references to color in this figure legend, the reader is referred to the free web version of this article.)

In the following example, we give an example for an anti-Salkowski curve which is Mannheim curve. This example is new in the literature.

Example 3. Let us consider the anti-Salkowski curve in $\mathbb{E}^{3}$ given by

$$
\beta(s)=\left(\begin{array}{l}
\frac{\left(24-s^{2}\right)^{3 / 2}\left(-144-9 s^{2}+5 s^{4}\right)}{37800}, \\
\frac{s\left(7560+1260 s^{2}-189 s^{4}+5 s^{6}\right)}{37800}, \\
-\frac{6}{5}\left(-2 \arcsin \left(\frac{\sqrt{24-s^{2}}}{2 \sqrt{6}}\right)+\sin \left(2 \arcsin \left(\frac{\sqrt{24-s^{2}}}{2 \sqrt{6}}\right)\right)\right)
\end{array}\right)
$$


with the curvatures $\kappa_{1}=\frac{s}{\sqrt{24-s^{2}}}$ and $\kappa_{2}=1$ and the Frenet frame as

$$
\begin{aligned}
T & =\left(\frac{-s^{3} \sqrt{24-s^{2}}\left(s^{2}-15\right)}{1080}, \frac{216+108 s^{2}-27 s^{4}+s^{6}}{1080}, \frac{-\sqrt{24-s^{2}}}{5}\right) \\
N & =\left(s-\frac{s^{3}}{6}+\frac{s^{5}}{180}, \frac{\sqrt{24-s^{2}}\left(36-18 s^{2}+s^{4}\right)}{180}, \frac{1}{5}\right) \\
B & =\left(1-\frac{s^{2}\left(540-45 s^{2}+s^{4}\right)}{1080}, \frac{s\left(24-s^{2}\right)^{3 / 2}\left(s^{2}-9\right)}{1080},-\frac{s}{5}\right) .
\end{aligned}
$$

If we take $v_{0}=0$ in Example 1, we obtain the Mannheim partner curve $\beta^{*}$ as follows

$$
\beta^{*}(s)=\left(\begin{array}{l}
\frac{\sqrt{24-s^{2}}\left(-4608+2004 s^{2}-178 s^{4}+5 s^{6}\right)}{50400}, \\
\frac{s\left(20160-3780 s^{2}+238 s^{4}-5 s^{6}\right)}{50400}, \\
-\frac{3}{10}\left(s \sqrt{24-s^{2}}-8 \operatorname{cosec}^{-1}\left(\frac{2 \sqrt{6}}{\sqrt{24-s^{2}}}\right)\right)
\end{array}\right),
$$

with the curvatures

$$
\kappa_{1}^{*}=\frac{2 \sqrt{6}}{3\left(16-s^{2}\right)} \quad \text { and } \quad \kappa_{2}^{*}=\frac{8}{16-s^{2}}
$$

the Frenet frame as

$$
\begin{aligned}
T^{*} & =\left(\frac{s\left(180-30 s^{2}+s^{4}\right)}{360 \sqrt{6}}, \frac{\sqrt{24-s^{2}}\left(36-18 s^{2}+s^{4}\right)}{360 \sqrt{6}},-\frac{2 \sqrt{6}}{5}\right), \\
N^{*} & =\left(\frac{\sqrt{24-s^{2}}\left(36-18 s^{2}+s^{4}\right)}{72 \sqrt{6}},-\frac{s\left(180-30 s^{2}+s^{4}\right)}{72 \sqrt{6}}, 0\right), \\
B^{*} & =\left(-s+\frac{s^{3}}{6}-\frac{s^{5}}{180},-\frac{\sqrt{24-s^{2}}\left(36-18 s^{2}+s^{4}\right)}{180},-\frac{1}{5}\right) .
\end{aligned}
$$

It can be easily obtained $N=-B^{*}$ which implies that $\beta$ is a Mannheim curve whose Mannheim partner curve is $\beta^{*}$. Here $\beta^{*}$ is a general helix.

Example 4. Let us consider the curve in $\mathbb{E}^{3}$ given by

$$
\beta(s)=\left(\begin{array}{l}
\frac{-3}{\sqrt{2}} \cos (\sqrt{2} s) \sin s+2 \cos s \sin (\sqrt{2} s) \\
\frac{-3}{\sqrt{2}} \sin (\sqrt{2} s) \sin s-2 \cos s \cos (\sqrt{2} s), \\
\frac{1}{\sqrt{2}} \sin s
\end{array}\right)
$$

with the curvatures $\kappa_{1}=\sin s$ and $\kappa_{2}=\cos s$ and the Frenet frame as

$$
\begin{aligned}
& T=\left(\begin{array}{c}
\frac{1}{\sqrt{2}} \cos (\sqrt{2} s) \cos s+\sin (\sqrt{2} s) \sin s, \\
\frac{1}{\sqrt{2}} \sin (\sqrt{2} s) \cos s-\cos (\sqrt{2} s) \sin s, \\
\frac{1}{\sqrt{2}} \cos (s)
\end{array}\right), \\
& N=\left(\frac{\cos (\sqrt{2} s)}{\sqrt{2}}, \frac{\sin (\sqrt{2} s)}{\sqrt{2}},-\frac{1}{\sqrt{2}}\right),
\end{aligned}
$$



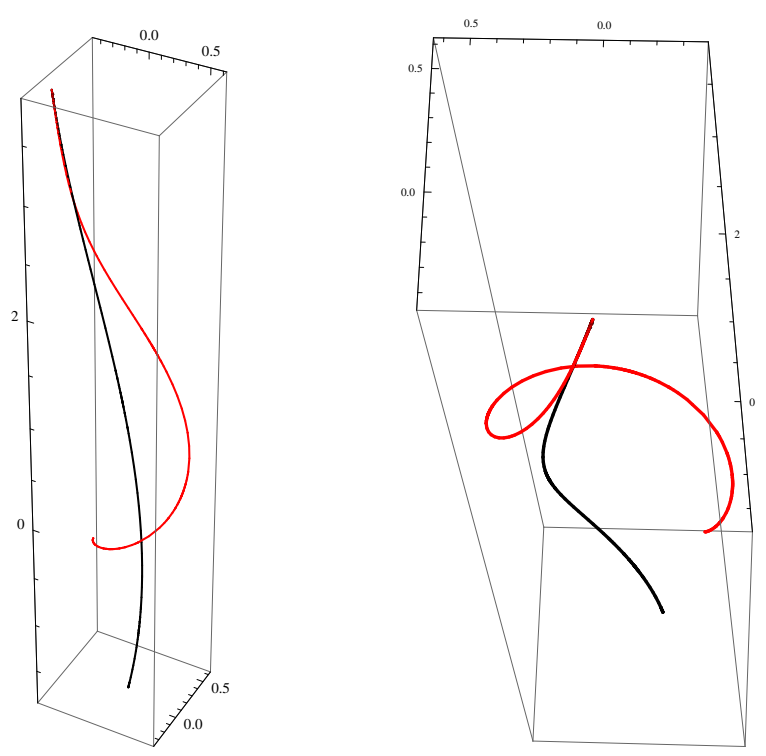

Figure 2: The figure contains same graphics from two different aspects. The red graphic is $\beta$ and the black graphic is $\beta^{*}$ in Example 3. (For interpretation of the references to color in this figure legend, the reader is referred to the free web version of this article.)

$$
B=\left(\begin{array}{l}
\frac{1}{\sqrt{2}} \cos (\sqrt{2} s) \sin (s)-\cos (s) \sin (\sqrt{2} s), \\
\frac{1}{\sqrt{2}} \sin (\sqrt{2} s) \sin (s)+\cos (s) \cos (\sqrt{2} s), \\
\frac{1}{\sqrt{2}} \sin (s)
\end{array}\right) .
$$

It can be easily obtained that

$$
\sigma=\left(\frac{\kappa_{2}}{\kappa_{1}}\right)^{\prime} \frac{\kappa_{1}^{2}}{\left(\kappa_{1}^{2}+\kappa_{2}^{2}\right)^{3 / 2}}=1 .
$$

So $\beta$ is a slant helix. If we take $v_{0}=0$ in Example 1, we obtain the Mannheim partner curve $\beta^{*}$ as follows

$$
\beta^{*}(s)=\left(\begin{array}{l}
-\sqrt{2} \cos (\sqrt{2} s) \sin s+2 \sin (\sqrt{2} s) \cos s, \\
-2 \cos (\sqrt{2} s) \cos s-\sqrt{2} \sin (\sqrt{2} s) \sin s, \\
\sqrt{2} \sin s
\end{array}\right)
$$

with the curvatures

$$
\kappa_{1}^{*}=\kappa_{2}^{*}=\frac{\sec s}{2}
$$

and the Frenet frame as

$$
\begin{aligned}
& T^{*}=\left(\frac{\cos (\sqrt{2} s)}{\sqrt{2}}, \frac{\sin (\sqrt{2} s)}{\sqrt{2}}, \frac{1}{\sqrt{2}}\right), \\
& N^{*}=(-\sin (\sqrt{2} s), \cos (\sqrt{2} s), 0), \\
& B^{*}=\left(-\frac{\cos (\sqrt{2} s)}{\sqrt{2}},-\frac{\sin (\sqrt{2} s)}{\sqrt{2}}, \frac{1}{\sqrt{2}}\right) .
\end{aligned}
$$


It can be easily obtained that $N=-B^{*}$ which implies that $\beta$ is a Mannheim curve whose Mannheim partner curve is $\beta^{*}$. Here $\beta^{*}$ is a general helix.
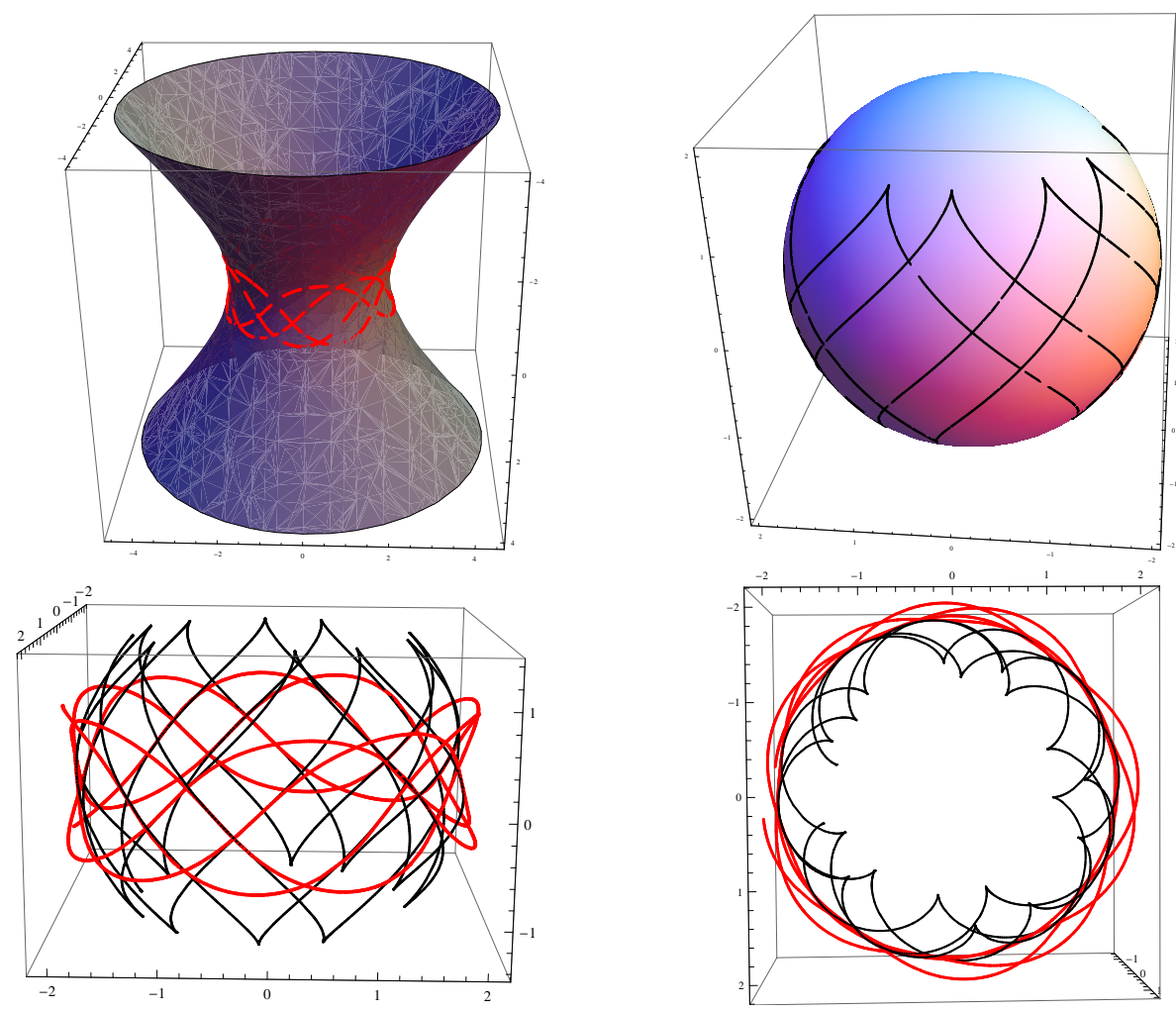

Figure 3: The figure contains same graphics from two different aspects. The red graphic is $\beta$ (lying on the hyperboloid $x^{2}+y^{2}-z^{2}=4$ ) and the black graphic is $\beta^{*}$ (lying on the sphere $x^{2}+y^{2}+z^{2}=4$ ) in Example 4. (For interpretation of the references to color in this figure legend, the reader is referred to the free web version of this article.)

Now we give a method to obtain anti-Salkowski curve in $\mathbb{E}^{3}$. Let $\beta: I \subset \mathbb{R} \rightarrow \mathbb{E}^{3}$ be a Mannheim curve except slant helices with the curvatures $\kappa_{1}, \kappa_{2}$ and the curve $\beta^{*}$ be a Mannheim partner curve of $\beta$ with the curvatures $\kappa_{1}^{*}, \kappa_{2}^{*}$. Assume that $\beta^{*}$ is a anti-Salkowski curve. Then from (2.18), for a nonzero constant $c$, we have

$$
\kappa_{2}^{*}=\frac{m_{1} \sqrt{\kappa_{1}^{2}+\kappa_{2}^{2}}}{f^{\prime}}=c \quad \text { or } \quad c f^{\prime}=m_{1} \sqrt{\kappa_{1}^{2}+\kappa_{2}^{2}}=\frac{c m_{1}\left(w^{\prime}+v \kappa_{2}\right) \sqrt{\kappa_{1}^{2}+\kappa_{2}^{2}}}{\kappa_{1}}
$$

which implies with (2.4) and (2.10) for $v=0$ that

$$
u=-\frac{\frac{\kappa_{2}}{\kappa_{1}}}{\left(\frac{\kappa_{2}}{\kappa_{1}}\right)^{\prime}} \quad \text { and } \quad w=-\frac{1}{\left(\frac{\kappa_{2}}{\kappa_{1}}\right)^{\prime}}=\frac{1}{c} \int \kappa_{1} d s .
$$

So we can give the following corollary. 
Corollary 2.5. Let $\beta: I \subset \mathbb{R} \rightarrow \mathbb{E}^{3}$ be a Mannheim curve except slant helices with the curvatures $\kappa_{1}, \kappa_{2}$ and the curve $\beta^{*}$ be a Mannheim partner curve of $\beta$ with the curvatures $\kappa_{1}^{*}, \kappa_{2}^{*}$ as

$$
\beta^{*}=\beta-\frac{\frac{\kappa_{2}}{\kappa_{1}}}{\left(\frac{\kappa_{2}}{\kappa_{1}}\right)^{\prime}} T-\frac{1}{\left(\frac{\kappa_{2}}{\kappa_{1}}\right)^{\prime}} B
$$

where

$$
\kappa_{2}=-c \kappa_{1} \int \frac{1}{\int \kappa_{1} d s} d s
$$

Then $\beta^{*}$ is an anti-Salkowski curve with $\kappa_{2}^{*}=c$ where $c \in \mathbb{R} /\{0\}$.

Example 5. If we take $\kappa_{1}=\cos s, \kappa_{2}=\cos s \ln \left(\cot \left(\frac{s}{2}\right)\right)$ and $c=1$, the condition of Corollary 2.5 is satisfied. So for the curve $\beta$ with $\kappa_{1}=\cos s, \kappa_{2}=\cos s \ln \left(\cot \left(\frac{s}{2}\right)\right)$, we obtain

$$
\beta^{*}=\beta-\sin s \ln \left(\tan \left(\frac{s}{2}\right)\right) T+\sin s B
$$

which is an anti-Salkowski curve with $\kappa_{2}^{*}=1$.

Similarly, we give a method to obtain Salkowski curve in $\mathbb{E}^{3}$. Let $\beta: I \subset \mathbb{R} \rightarrow \mathbb{E}^{3}$ be a Mannheim curve except slant helices with the curvatures $\kappa_{1}, \kappa_{2}$ and the curve $\beta^{*}$ be a Mannheim partner curve of $\beta$ with the curvatures $\kappa_{1}^{*}, \kappa_{2}^{*}$. Assume that $\beta^{*}$ is a Salkowski curve. Then from (2.18), for a nonzero constant $c$, we have

$$
\kappa_{1}^{*}=\frac{-m_{2} \kappa_{1}^{2}\left(\frac{\kappa_{2}}{\kappa_{1}}\right)^{\prime}}{f^{\prime}\left(\kappa_{1}^{2}+\kappa_{2}^{2}\right)}=c \quad \text { or } \quad f^{\prime}=-\frac{m_{2} \kappa_{1}^{2}\left(\frac{\kappa_{2}}{\kappa_{1}}\right)^{\prime}}{c\left(\kappa_{1}^{2}+\kappa_{2}^{2}\right)}=\frac{m_{1}\left(w^{\prime}+v \kappa_{2}\right) \sqrt{\kappa_{1}^{2}+\kappa_{2}^{2}}}{\kappa_{1}} .
$$

Then we find

$$
w^{\prime}+v \kappa_{2}=-\frac{m_{1} m_{2}}{c}\left(\frac{\kappa_{2}}{\kappa_{1}}\right)^{\prime} \frac{\kappa_{1}^{3}}{\left(\kappa_{1}^{2}+\kappa_{2}^{2}\right)^{3 / 2}}=-\frac{m_{1} m_{2}}{c}\left(\frac{\kappa_{2}}{\kappa_{1}}\right)^{\prime} \frac{1}{\left(1+\left(\frac{\kappa_{2}}{\kappa_{1}}\right)^{2}\right)^{3 / 2}}
$$

which implies with (2.4) and (2.10) for $v=0$ that

$$
w^{\prime}=-\frac{m_{1} m_{2}}{c}\left(\frac{\kappa_{2}}{\kappa_{1}}\right)^{\prime} \frac{1}{\left(1+\left(\frac{\kappa_{2}}{\kappa_{1}}\right)^{2}\right)^{3 / 2}}, \quad u=w \frac{\kappa_{2}}{\kappa_{1}} \quad \text { and } \quad 1+u^{\prime}=w^{\prime} \frac{\kappa_{2}}{\kappa_{1}} .
$$

Then, we have

$$
u^{\prime}=w^{\prime} \frac{\kappa_{2}}{\kappa_{1}}+w\left(\frac{\kappa_{2}}{\kappa_{1}}\right)^{\prime} \text { and } u^{\prime}=w^{\prime} \frac{\kappa_{2}}{\kappa_{1}}-1
$$

which causes that

$$
u=-\frac{\frac{\kappa_{2}}{\kappa_{1}}}{\left(\frac{\kappa_{2}}{\kappa_{1}}\right)^{\prime}} \text { and } w=-\frac{1}{\left(\frac{\kappa_{2}}{\kappa_{1}}\right)^{\prime}}=-\frac{m_{1} m_{2}}{c} \int\left(\frac{\kappa_{2}}{\kappa_{1}}\right)^{\prime} \frac{1}{\left(1+\left(\frac{\kappa_{2}}{\kappa_{1}}\right)^{2}\right)^{3 / 2}} d s .
$$

Here

$$
-\frac{1}{\left(\frac{\kappa_{2}}{\kappa_{1}}\right)^{\prime}}=-\frac{m_{1} m_{2} \frac{\kappa_{2}}{\kappa_{1}}}{c \sqrt{1+\left(\frac{\kappa_{2}}{\kappa_{1}}\right)^{2}}}+c_{0}
$$


or

$$
1=\frac{m_{1} m_{2}}{c} \frac{\frac{\kappa_{2}}{\kappa_{1}}\left(\frac{\kappa_{2}}{\kappa_{1}}\right)^{\prime}}{\sqrt{1+\left(\frac{\kappa_{2}}{\kappa_{1}}\right)^{2}}}+c_{0}\left(\frac{\kappa_{2}}{\kappa_{1}}\right)^{\prime}
$$

Integrating both side with respect to $s$, we find

$$
s+c_{1}=\frac{m_{1} m_{2}}{c} \sqrt{1+\left(\frac{\kappa_{2}}{\kappa_{1}}\right)^{2}}+c_{0}\left(\frac{\kappa_{2}}{\kappa_{1}}\right) .
$$

So we can give the following corollary.

Corollary 2.6. Let $\beta: I \subset \mathbb{R} \rightarrow \mathbb{E}^{3}$ be a Mannheim curve except slant helices with the curvatures $\kappa_{1}, \kappa_{2}$ and the curve $\beta^{*}$ be a Mannheim partner curve of $\beta$ with the curvatures $\kappa_{1}^{*}, \kappa_{2}^{*}$ as

$$
\beta^{*}=\beta-\frac{\frac{\kappa_{2}}{\kappa_{1}}}{\left(\frac{\kappa_{2}}{\kappa_{1}}\right)^{\prime}} T-\frac{1}{\left(\frac{\kappa_{2}}{\kappa_{1}}\right)^{\prime}} B .
$$

Then $\beta^{*}$ is a Salkowski curve with $\kappa_{1}^{*}=$ c satisfying

$$
s+c_{1}=\frac{m_{1} m_{2}}{c} \sqrt{1+\left(\frac{\kappa_{2}}{\kappa_{1}}\right)^{2}}+c_{0}\left(\frac{\kappa_{2}}{\kappa_{1}}\right) .
$$

where $c \in \mathbb{R} /\{0\}$ and $c_{0}, c_{1} \in \mathbb{R}$.

Example 6. If we take $\kappa_{1}=s, \kappa_{2}=s \sqrt{s^{2}-1}, c=m_{1}=m_{2}=1$ and $c_{0}=c_{1}=0$, the condition of Corollary 2.6 is satisfied. So for the curve $\beta$ with $\kappa_{1}=s$ and $\kappa_{2}=s \sqrt{s^{2}-1}$, we obtain

$$
\beta^{*}=\beta-\frac{s^{2}-1}{s} T-\frac{\sqrt{s^{2}-1}}{s} B
$$

which is a Salkowski curve with $\kappa_{1}^{*}=1$.

In the following theorem, we characterize the Mannheim partner curves in $\mathbb{E}^{3}$. So we will give the necesarry and sufficient conditions for a curve to be a Mannheim partner curve.

Theorem 2.7. Let $\beta^{*}: I^{*} \rightarrow \mathbb{E}^{3}$ be a curve with the Frenet frame $\left\{T^{*}, N^{*}, B^{*}\right\}$ and the non-zero curvatures $\kappa_{1}^{*}, \kappa_{2}^{*}$. Then $\beta^{*}$ is a Mannheim partner curve of a certain Mannheim curve if and only if there exist differentiable functions $a, b, c$ and $\lambda$ satisfying

$$
\begin{gathered}
\dot{c}+b \kappa_{2}^{*}=0 \quad 1+\dot{a}-b \kappa_{1}^{*} \neq 0 \quad \dot{\lambda}=-\kappa_{1}^{*}\left(1+\lambda^{2}\right) \\
a \kappa_{1}^{*}+\dot{b}-c \kappa_{2}^{*}=\lambda\left(1+\dot{a}-b \kappa_{1}^{*}\right) .
\end{gathered}
$$

Here" " means that derivative with respect to $s^{*}$.

Proof. Assume that $\beta$ is a Mannheim curve parametrized by arc-length $s$ with non-zero curvatures $\kappa_{1}, \kappa_{2}$ and the curve $\beta^{*}$ is the Mannheim partner curve of the curve $\beta$ parametrized by with arclength or pseudo arc $s^{*}$. Then, we can write the curve $\beta$ as

$$
\beta(s)=\beta\left(h\left(s^{*}\right)\right)=\beta^{*}\left(s^{*}\right)+a(s) T^{*}\left(s^{*}\right)+b(s) N^{*}\left(s^{*}\right)+c(s) B^{*}\left(s^{*}\right)
$$


for all $s^{*} \in I^{*}$ where $a(s), b(s)$ and $c(s)$ are differentiable functions on $I^{*}$. Differentiating (2.21) with respect to $s^{*}$, we get

$$
T h^{\prime}=\left(1+\dot{a}-b \kappa_{1}^{*}\right) T^{*}+\left(a \kappa_{1}^{*}+\dot{b}-c \kappa_{2}^{*}\right) N^{*}+\left(\dot{c}+b \kappa_{2}^{*}\right) B^{*} .
$$

By taking the scalar product of $(2.22)$ with $B^{*}$, we have

$$
\dot{c}+b \kappa_{2}^{*}=0 .
$$

Substituting (2.23) in (2.22), we find

$$
T h^{\prime}=\left(1+\dot{a}-b \kappa_{1}^{*}\right) T^{*}+\left(a \kappa_{1}^{*}+\dot{b}-c \kappa_{2}^{*}\right) N^{*} .
$$

By taking the scalar product of (2.24) with itself, we obtain

$$
\left(h^{\prime}\right)^{2}=\left(1+\dot{a}-b \kappa_{1}^{*}\right)^{2}+\left(a \kappa_{1}^{*}+\dot{b}-c \kappa_{2}^{*}\right)^{2} .
$$

If we denote

$$
\delta=\frac{1+\dot{a}-b \kappa_{1}^{*}}{h^{\prime}} \text { and } \gamma=\frac{a \kappa_{1}^{*}+\dot{b}-c \kappa_{2}^{*}}{h^{\prime}}
$$

we get

$$
T=\delta T^{*}+\gamma N^{*} .
$$

Assume that $\delta=0$. Then we have $T=\gamma N^{*}$. Differentiating it with respect to $s^{*}$, we find

$$
h^{\prime} \kappa_{1} N=-\gamma \kappa_{1}^{*} T^{*}+\dot{\gamma} N^{*}+\gamma \kappa_{2}^{*} B^{*},
$$

which implies that $\kappa_{1}^{*}=0$. This is a contradiction. Thus $\delta \neq 0$. Similarly we can find $\gamma \neq 0$. So we obtain

$$
1+\dot{a}-b \kappa_{1}^{*} \neq 0
$$

and

$$
a \kappa_{1}^{*}+\dot{b}-c \kappa_{2}^{*}=\lambda\left(1+\dot{a}-b \kappa_{1}^{*}\right)
$$

where $\lambda=\gamma / \delta$. Differentiating (2.27) with respect to $s^{*}$, we find

$$
h^{\prime} \kappa_{1} N=\left(\dot{\delta}-\gamma \kappa_{1}^{*}\right) T^{*}+\left(\delta \kappa_{1}^{*}+\dot{\gamma}\right) N^{*}+\gamma \kappa_{2}^{*} B^{*} .
$$

By taking the scalar product of $(2.28)$ with $B^{*}$, we get

$$
\dot{\delta}-\gamma \kappa_{1}^{*}=0 \quad \text { and } \quad \delta \kappa_{1}^{*}+\dot{\gamma}=0
$$

which implies that $\dot{\lambda}=-\kappa_{1}^{*}\left(1+\lambda^{2}\right)$.

Conversely, assume that $\beta^{*}$ is a curve parametrized by arc-length $s^{*}$ with non-zero curvatures $\kappa_{1}^{*}, \kappa_{2}^{*}$ and the conditions of (2.19) and (2.20) hold for differentiable functions $a, b, c$ and $\lambda$. Then, we can define a curve $\beta$ as

$$
\beta\left(s^{*}\right)=\beta^{*}\left(s^{*}\right)+a(s) T^{*}\left(s^{*}\right)+b(s) N^{*}\left(s^{*}\right)+c(s) B^{*}\left(s^{*}\right)
$$


Differentiating (2.29) with respect to $s^{*}$, we find

$$
\frac{d \beta}{d s^{*}}=\left(1+\dot{a}-b \kappa_{1}^{*}\right) T^{*}+\left(a \kappa_{1}^{*}+\dot{b}-c \kappa_{2}^{*}\right) N^{*} .
$$

which leads to that

$$
h^{\prime}=\sqrt{\left\langle\frac{d \beta}{d s^{*}}, \frac{d \beta}{d s^{*}}\right\rangle}=m_{1}\left(1+\dot{a}-b \kappa_{1}^{*}\right) \sqrt{1+\lambda^{2}},
$$

where $m_{1}=\operatorname{sgn}\left(1+\dot{a}-b \kappa_{1}^{*}\right)$. Rewriting (2.30), we obtain

$$
T=\frac{m_{1}}{\sqrt{1+\lambda^{2}}}\left(T^{*}+\lambda N^{*}\right), \quad g(T, T)=1 .
$$

Differentiating (2.32) with respect to $s^{*}$, we find

$$
\frac{d T}{d s}=\frac{m_{1} \lambda \kappa_{2}^{*}}{h^{\prime} \sqrt{1+\lambda^{2}}} B^{*}
$$

which cause that

$$
\kappa_{1}=\left\|\frac{d T}{d s}\right\|=\frac{m_{2} m_{3} \lambda \kappa_{2}^{*}}{h^{\prime} \sqrt{1+\lambda^{2}}},
$$

where $m_{2}=\operatorname{sgn}(\lambda)$ and $m_{3}=\operatorname{sgn}\left(\kappa_{2}^{*}\right)$. Now, we can find $N$ as

$$
N=m_{1} m_{2} m_{3} B^{*}, \quad g(N, N)=1 .
$$

Now, we define $B$ as

$$
B=T \times N=\frac{m_{2} m_{3}}{\sqrt{1+\lambda^{2}}}\left(\lambda T^{*}-N^{*}\right), \quad g(B, B)=1 .
$$

Lastly we find

$$
\kappa_{2}=\left\langle\frac{d N}{d s}, B\right\rangle=\frac{m_{1} \kappa_{2}^{*}}{h^{\prime} \sqrt{1+\lambda^{2}}} \neq 0 .
$$

Then $\beta^{*}$ is a Mannheim partner curve of $\beta$.

If we take $a=b=0$ in Theorem 2.7, we have the conditions of classical Mannheim partner curves in the literature with

$$
\beta\left(s^{*}\right)=\beta^{*}\left(s^{*}\right)+c(s) B^{*}\left(s^{*}\right)
$$

Also we have

$$
\dot{c}=0, \quad \dot{\lambda}=-\kappa_{1}^{*}\left(1+\lambda^{2}\right), \quad-c \kappa_{2}^{*}=\lambda .
$$

So we get

$$
\frac{d \kappa_{2}^{*}}{d s^{*}}=\frac{\kappa_{1}^{*}}{c}\left(1+c^{2}\left(\kappa_{2}^{*}\right)^{2}\right) .
$$

Thus we can give the following corollary. The following corollary can be seen in [6].

Corollary 2.8. Let $\beta^{*}: I^{*} \rightarrow \mathbb{E}^{3}$ be a curve with the Frenet frame $\left\{T^{*}, N^{*}, B^{*}\right\}$ and the non-zero curvatures $\kappa_{1}^{*}, \kappa_{2}^{*}$. Then $\beta^{*}$ is a Mannheim partner curve of a certain Mannheim curve given by

$$
\beta\left(s^{*}\right)=\beta^{*}\left(s^{*}\right)+c(s) B^{*}\left(s^{*}\right)
$$

if and only if there exist real number c satisfying

$$
\frac{d \kappa_{2}^{*}}{d s^{*}}=\frac{\kappa_{1}^{*}}{c}\left(1+c^{2}\left(\kappa_{2}^{*}\right)^{2}\right) .
$$




\section{References}

[1] A. T. Ali, Position vectors of slant helices in Euclidean 3-space, Journal of the Egyptian Mathematical Society, 20 (2012), 1-6.

[2] C. Camciand A. Uçum and K. İlarslan, A new approach to Bertrand Curves in Euclidean 3-space, J. Geom., 111 (2020), 49.

[3] S. Izumiya and N. Takeuchi, New special curves and developable surfaces, Turk. J. Math., 28 (2004), 531-537.

[4] W. Kuhnel, Differential geometry: curves-surfaces-manifolds, Braunschweig: Wiesbaden, 1999.

[5] M. A. Lancret, Mémoire sur les courbes à double courbure Mémoires présentés à l'Institut1, (1806), 416-454.

[6] H. Liu and F. Wang, Mannheim partner curves in 3-space, J. Geom., 88 (2008), 120-126.

[7] J. Miller, Note on Tortuous Curves, Proceedings of the Edinburgh Mathematical Society, 24 (1905), 51-55.

[8] J. Monterde, Salkowski curves revisted: A family of curves with constant curvature and non-constant torsion, Comput. Aided Geomet. Design, 26 (2009), 271-278.

[9] E. Salkowski, Zur transformation von raumkurven Mathematische Annalen, 66 (1909), 517-557.

[10] D. J. Struik, Lectures on classical differential geometry, Dover publications, New York, 1961.

[11] O. Tigano, Sulla determinazione delle curve di Mannheim, Matematiche Catania, 3 (1948), 25-29.

Ali Uçum Kırıkkale University, Faculty of Sciences and Arts, Department of Mathematics, Kırıkkale-Turkey

E-mail: aliucum05@gmail.com

Çetin Camcı Department of Mathematics, Faculty of Sciences and Arts, Onsekiz Mart University, Çanakkale, TURKEY

E-mail: ccamci@comu.edu.tr

Kazım İlarslan Kırıkkale University, Faculty of Sciences and Arts, Department of Mathematics, Kırıkkale-Turkey

E-mail: kilarslan@yahoo.com 